\title{
ON FIBER SPACES
}

\author{
BY \\ EDWARD FADELL( $\left({ }^{1}\right)$
}

1. Introduction. The first part of this paper is devoted to setting forth for future reference some results on fiber spaces in the sense of Hurewicz [1] and also to illustrate techniques associated with the use of "lifting functions." The second part ( $\$ 5$ ), which was motivated by what preceded, is devoted to exploring a definition of fiber space which is invariant under fiber-homotopy equivalence [2]; i.e., if $(X, B, p)$ and $(X, B, q)$ are fiber-homotopy equivalent triples, then if one is a fiber space so is the other. Of course, this is not true for fiber spaces in the sense of Hurewicz [1] or Serre [3].

2. Preliminaries. For the convenience of the reader, we recall the definition of fiber space [1] employed in $\S 3$. If $p: X \rightarrow B$ is a map (=continuous function), let $\Omega_{p}$ denote the subset of $X \times B^{I}$ consisting of ordered pairs $(x, \omega)$ such that $p(x)=\omega(0)$. Then, we have a natural map $\tilde{p}: X^{I} \rightarrow \Omega_{p}$ given by $\tilde{p}(\alpha)=(\alpha(0), p \alpha) .(X, B, p)$ is called a fiber space (in the sense of Hurewicz) if $\tilde{p}$ admits a cross section $\lambda$, i.e., a map $\lambda: \Omega_{p} \rightarrow X^{I}$ such that $\tilde{p} \lambda=1$. Such a map $\lambda$ is called a lifting function. It is easy to see that $(X, B, p)$ is a fiber space if and only if the Covering Homotopy Theorem holds for $(X, B, p)$ with respect to every topological space. A lifting function $\lambda$ is called regular if $\lambda(x, \omega)$ is a constant path whenever $\omega$ is. If $(X, B, p)$ admits a regular lifting function it is called a regular fiber space. Every fiber space $(X, B, p)$ with $B$ metric is regular [1]. We note also that fiber spaces in the sense of Hurewicz-Steenrod and $\mathrm{Hu}$ (see [1]) are fiber spaces in the above sense if the base space is paracompact and hence subsequent results will apply in these situations.

In the sequel it will be convenient to obtain a local product representation of fiber spaces up to fiber-homotopy equivalence. This we will be able to do for fiber spaces $(X, B, p)$ where $B$ satisfies a weak locally contractible condition.

Definition. A space $B$ is weakly locally contractible (wlc) if for every $b_{0} \in B$, there exists an open set $U$ containing $b_{0}$ which is contractible to $b_{0}$ in $B$, i.e., there exists a homotopy $H: U \times I \rightarrow B$ such that $H_{0}=1$ and $H_{1}=b_{0}$.

We note that any contractible space is wlc and furthermore, we show below that wlc is a homotopy type invariant. These properties justify the use of the property wlc rather than the perhaps more natural property "locally contractible."

Proposition. wlc is a homotopy type invariant.

Received by the editors March 14, 1957.

(1) This paper was sponsored in part by the National Science Foundation under Grant NSF G-4223. 
Proof. Suppose $\psi: X \rightleftarrows Y: \phi$ is a homotopy equivalence and $X$ is wlc. Take $y_{0} \in Y$ and set $x_{0}=\phi\left(y_{0}\right)$. Let $U$ denote an open set in $X$ containing $x_{0}$ and $H: U \times I \rightarrow X$ a homotopy such that $H_{0}=1$ and $H_{1}=x_{0}$. Let $\Phi: Y \times I \rightarrow Y$ denote a homotopy such that $\Phi_{0}=1$ and $\Phi_{1}=\psi \phi$. Let $V=\phi^{-1}(U)$ and define a homotopy $G: V \times I \rightarrow Y$ as follows

$$
G(y, t)=\left\{\begin{array}{l}
\Phi(y, 2 t), \quad 0 \leqq t \leqq 1 / 2, \\
\psi[H(\phi(y), 2 t-1)], \quad 1 / 2 \leqq t \leqq 1 .
\end{array}\right.
$$

Then, it is easy to see that $G_{0}=1$ and $G_{1}=\psi\left(x_{0}\right)=\psi \phi\left(y_{0}\right)$. Since $\psi \phi\left(y_{0}\right)$ and $y_{0}$ lie in the same arc component of $Y$, our proof is complete.

REMARK. Actually what we showed in the above is that a space dominated by a wlc space is wlc.

3. Basic theorems in fiber spaces. Let $(X, B, p)$ denote a fiber space and $\lambda$ a lifting function. $\lambda$ induces a map $\tilde{\lambda}: X^{I} \rightarrow X^{I}$ as follows.

$$
\tilde{\lambda}(\alpha)=\lambda[\alpha(0), p \alpha], \quad \alpha \in X^{I} .
$$

The following result is basic.

Proposition 1. $\tilde{\lambda} \sim 1$ preserving projections, i.e., there exists a homotopy $H: X^{I} \times I \rightarrow X^{I}$ such that $H_{0}=1, H_{1}=\tilde{\lambda}$ and $p H(\alpha, s)(t)=p \alpha(t)$ for $\alpha \in X^{I}$; $s, t \in I$.

Proof. For $\alpha \in X^{I}, s \in I$, let $\alpha_{s} \in X^{I}$ denote the path given by

$$
\alpha_{\varepsilon}(t)= \begin{cases}\alpha(t), & 0 \leqq t \leqq s, \\ \alpha(s), & s \leqq t \leqq 1 .\end{cases}
$$

Set $\omega=p \alpha$ and let $\omega^{1-s}$ denote the path

$$
\omega^{1-s}(t)= \begin{cases}\omega(s+t), & 0 \leqq t \leqq 1-s \\ \omega(1), & 1-s \leqq t \leqq 1\end{cases}
$$

Then, the required homotopy $H: X^{I} \times I \rightarrow X^{I}$ is obtained by setting

$$
H(\alpha, s)(t)=\left\{\begin{array}{l}
\alpha_{s}(t), \quad 0 \leqq t \leqq s, \\
\lambda\left[\alpha(s), \omega^{1-s}\right](t-s), \quad s \leqq t \leqq 1 .
\end{array}\right.
$$

Proposition 2. If $(X, B, p)$ is a fiber space and if $B$ is 0-connected, then all the fibers $p^{-1}(b), b \in B$ have the same homotopy type.

Proof. Take $b$ and $b^{\prime}$ in $B$ and let $F=p^{-1}(b), F^{\prime}=p^{-1}\left(b^{\prime}\right)$. Let $\omega$ denote a path from $b$ to $b^{\prime}$, and $\omega^{*}$ the inverse of $\omega$, i.e., $\omega^{*}(t)=\omega(1-t)$. We set up a homotopy equivalence $\phi: F \rightleftarrows F^{\prime}: \psi$ as follows. If $\lambda$ is a lifting function for $(X, B, p)$, set

and

$$
\phi(x)=\lambda[x, \omega](1), \quad x \in F
$$




$$
\psi\left(x^{\prime}\right)=\lambda\left[x^{\prime}, \omega^{*}\right](1), \quad x^{\prime} \in F^{\prime} .
$$

We show that $\psi \phi \sim 1$. That $\phi \psi \sim 1$ follows in a similar manner. Let $\tilde{\lambda}$ denote the map of Proposition 1 and let $H$ be a homotopy connecting 1 and $\tilde{\lambda}$, preserving projections. Define a homotopy $G: F \times I \rightarrow F$ as follows. Set

$$
G(x, t)=H\left[\lambda(x, \omega)^{*}, t\right](1)
$$

where $\lambda(x, \omega)^{*}(t)=\lambda(x, \omega)(1-t)$. Then, $G(x, 0)=x, G(x, 1)=\psi \phi(x)$ and since $H$ preserves projections $G(x, t) \in F$. Therefore, $\psi \phi \sim 1$ in $F$ and our result follows.

The next result is analogous to one given by G. S. Young [4] for fiber bundles.

Proposition 3. Let $(X, B, p)$ denote a fiber space with $X$ 0-connected. Then any two arc-components of a given fiber are of the same homotopy type.

Proof. Take $b \in B$ and let $C$ and $C^{\prime}$ denote two arc components of $p^{-1}(b)$. Fix $x_{0} \in C$ and $x_{0}^{\prime} \in C^{\prime}$ and let $\alpha$ denote a path in $X$ from $x_{0}$ to $x_{0}^{\prime}$. A homotopy equivalence $\phi: C \rightleftarrows C^{\prime}: \psi$ is set up as follows:

$$
\begin{aligned}
\phi(x) & =\lambda[x, \omega](1), & & x \in C, \\
\psi\left(x^{\prime}\right) & =\lambda\left[x^{\prime}, \omega^{*}\right](1), & & x^{\prime} \in C^{\prime}
\end{aligned}
$$

where $\lambda$ is a lifting function, $\omega=p \alpha$ and $\omega^{*}$ is the inverse of $\omega$. We first show that $\phi(x) \in C^{\prime}$, i.e., $\phi: C \rightarrow C^{\prime}$. It suffices to exhibit a path from $\phi(x)$ to $x_{0}^{\prime}$. Let $\beta$ denote a path from $x$ to $x_{0}$ and set

$$
\gamma(t)=\lambda[\beta(t), \omega](1) .
$$

Then, $\gamma$ is a path from $\phi(x)$ to $\phi\left(x_{0}\right)$. Since $\phi\left(x_{0}\right)$ is obtained by lifting the projection of $\alpha$ with initial point $\alpha(0)$, we may apply Proposition 1 and obtain a path from $\phi\left(x_{0}\right)$ to $x_{0}^{\prime}$. Therefore, we have the required path from $\phi(x)$ to $x_{0}^{\prime}$ and hence $\phi(C) \subseteq C^{\prime}$. Similarly $\psi\left(C^{\prime}\right) \subseteq C$. Now, making use of Proposition 1 in much the same manner as in the previous result, it is easy to see that $\phi$ and $\psi$ form a homotopy equivalence.

REMARK. As is well known, Propositions 2 and 3 are false for fiber spaces in the sense of Serre.

We review now the definition of fiber-homotopy equivalence. Two triples $(X, B, p),(Y, B, q)$ are fiber-homotopy equivalent if there exist fiber maps $\phi: X \rightleftarrows Y: \psi$ such that $\psi \phi \sim 1$ and $\phi \psi \sim 1$ with projection-preserving homotopies, e.g., if $H$ is a homotopy connecting $\psi \phi$ and $1, H$ is required to satisfy the condition

$$
p H(x, t)=p(x), \quad 0 \leqq t \leqq 1 .
$$

The pair $(\phi, \psi)$ is called a fiber-homotopy equivalence.

Proposition 4. If $(X, B, p)$ is a fiber space with $B$ wlc, then for each $b \in B$, 
there is an open set $U$ containing $b$ such that $\left(p^{-1}(U), U, p\right)$ is fiber-homotopy equivalent to $U \times F_{b}$, where $F_{b}=p^{-1}(b)$. In particular, if $B$ is contractible, $(X, B, p)$ is fiber-homotopy equivalent to a product space.

Proof. For $b_{0} \in B$, let $U$ denote an open set containing $b$ which can be contracted to $b_{0}$ in $B$; i.e., there exists a map $H: U \rightarrow B^{I}$ such that $H(b)(0)=b$, $H(b)(1)=b_{0}$. Let $\lambda$ denote a lifting function and define $\phi: p^{-1}(U) \rightarrow U \times F$ as follows:

$$
\phi(x)=(p(x), \lambda[x, H(p(x))](1)) .
$$

$\psi: U \times F \rightarrow p^{-1}(U)$ is defined by

$$
\psi(b, f)=\lambda\left[f, H(b)^{*}\right](1)
$$

where $H(b)^{*}$ is the inverse of the path $H(b)$. It is clear that $\xi \phi=p \psi$ where $\xi: U \times F \rightarrow U$ is the natural projection. Furthermore, applying Proposition 1 one sees that $\phi \psi \sim 1$ and $\psi \phi \sim 1$, preserving projections and hence $\left(p^{-1}(U)\right.$, $U, p)$ is fiber-homotopy equivalent to $(U \times F, U, \xi)$.

Combining Propositions 2 and 4 we obtain:

Proposition 5. If $(X, B, p)$ is a fiber space with $B$ wlc and 0-connected, then there exists a single space $F$ and a family $\left\{U_{\alpha}\right\}$ of open sets covering $B$ such that $\left(p^{-1}\left(U_{\alpha}\right), U_{\alpha}, p\right)$ is fiber-homotopy equivalent to the product $U_{\alpha} \times F$; i.e., $(X, B, p)$ is locally trivial-up to fiber-homotopy equivalence.

Remark. Propositions 4 and 5 are false for fiberings with base space not wlc (see $\$ 4$ ). Proposition 5 motivates the definition of fiber space we consider in $\$ 5$.

Proposition 6. Let $(X, B, p)$ denote a fiber space where $X$ is metric and $B$ is wlc and paracompact. Then $(X, B, p)$ is a regular fiber space.

Proof. Take $b \in B$. Then by Proposition 4 , there is an open set $U$ containing $b$ such that $\left(p^{-1}(U), U, p\right)$ is fiber-homotopy equivalent to $U \times F_{b}$. Therefore, $p \mid p^{-1}(U)$ admits a cross section and therefore $U$ may be imbedded in $X$. Hence $U$ is metric. Therefore, (see $\S 2)\left(p^{-1}(U), U, p\right)$ is a regular fiber space and $(X, B, p)$ is locally regular. Since $B$ is paracompact, we may apply the Uniformization Theorem in [1] and conclude that $(X, B, p)$ is a regular fiber space. Actually, once we know that $B$ is locally metric and paracompact, we could apply a theorem of Smirnov [5] and conclude that $B$ is metric. Whereupon, we may apply the result that a fiber space with metric base is regular.

Proposition 7. Let $(X, B, p)$ denote a fiber space and $F=p^{-1}(b), b \in B, a$ fiber which is contractible in $X$ to $x_{0} \in F$. Let $\Lambda$ denote the space of loops of $B$ based at $b$. Then $\Lambda$ dominates $F$.

Proof. Since $F$ is contractible in $X$, there is a map $C: F \rightarrow X^{I}$ such that 
$C(x)(0)=x_{0}$ and $C(x)(1)=x$, for $x \in F$. Let $\widetilde{F}_{0} \subseteq X^{I}$ denote the paths in $X$ emanating from $x_{0}$ and terminating in $F$. Then, $p: X \rightarrow B$ induces a map $\tilde{p}: \widetilde{F}_{0} \rightarrow \Lambda$ by setting $\tilde{p}(\alpha)(t)=p[\alpha(t)]$, for $\alpha \in \widetilde{F}_{0}$ and $t \in I$. Let $\phi: F \rightarrow \Lambda$ be the map $\tilde{p} C$. A map $\psi: \Lambda \rightarrow F$ is obtained as follows. For $\omega \in \Lambda$, set

$$
\psi(\omega)=\lambda\left(x_{0}, \omega\right)(1)
$$

where $\lambda$ is a lifting function. Let $H$ denote the homotopy of Proposition 1 connecting 1 and $\tilde{\lambda}$. Define $G: F \times I \rightarrow F$ by

$$
G(x, t)=H[C(x), t](1) .
$$

Then, $G(x, 0)=x$ and $G(x, 1)=\tilde{\lambda}(C(x))(1)=\lambda\left[x_{0}, \phi(x)\right](1)=\psi \phi(x)$. Therefore, $\psi \phi \sim 1$ and $\Lambda$ dominates $F$.

This result provides a necessary condition for the existence of fiberings with fibers contractible in the total space $\left({ }^{2}\right)$. We note further that if in Proposition 7 , we assume that $(X, B, p)$ is regular and that $F$ is contractible in $X$ relative to $x_{0}$, then in the above proof we would have the following. If $e$ is the constant loop at $b$, then $\phi\left(x_{0}\right)=e, \psi(e)=x_{0}$ and $\psi \phi \sim 1$ relative to $x_{0}$.

The next proposition is an analogue of Theorems 1.1 and 3.1 of $\mathrm{E}$. H. Spanier and J. H. C. Whitehead [6]. Although we are assuming a stronger covering homotopy theorem, no hypotheses (e.g., ANR, $C W$-complex) are made on the spaces involved. The proof is different and is based on Proposition 7 and the following lemma. We use the term $H$-space as given in [3] which is slightly more general than that used in [6].

Lemma. Let $\Lambda$ denote an $H$-space with homotopy-identity e. Suppose $F$ is a space and $\phi: F \rightleftarrows \Lambda: \psi$ are given maps such that

(1) there exists an element $x_{0} \in F$ such that $\phi\left(x_{0}\right)=e$,

(2) $\psi \phi \sim 1$ relative to $x_{0}$

Then $F$ is an $H$-space with $x_{0}$ as homotopy-identity.

The simple proof is left to the reader.

Proposition 8. Let $(X, B, p)$ denote a regular fiber space and $F=p^{-1}(b)$ a fiber. If $F$ is contractible in $X$ to a point $x_{0} \in F$ relative to $x_{0}$, then $F$ is an $H$ space.

Proof $\left(^{3}\right)$. This result is an immediate consequence of the remarks following Proposition 7 , the preceding lemma, and the fact that the loop space $\Lambda$ is an $H$-space.

REMARK. The binary operation in $F$ above is geometrically the following. Given two elements $x$ and $x^{\prime}$ in $F$, the contraction of $F$ in $X$ yields two paths in $X$ from $x_{0}$ to $x$ and $x^{\prime}$, respectively. These paths are then projected into $B$ and multiplied as loops. The product loop is then lifted with initial point $x_{0}$.

(2) It is not difficult to show that Proposition 7 is false for fiberings in the sense of Serre.

(3) E. H. Spanier informs me that a similar proof was communicated to him by J. P. Serre. 
The right hand end point of this path is the product of $x$ and $x^{\prime}$.

Our next proposition is a cross section theorem for fiber spaces. The classical cross-section theorem [7] is false for fiber spaces even when the spaces involved are separable metric. The hypothesis that the base space in wlc seems crucial (see $\$ 4$ ).

Proposition 9. Let $(X, B, p)$ denote a fiber space such that $X$ and $B$ are separable metric and $X$ is an $A N R$ (separable metric). If $B$ is 0-connected and wlc and the fibers are contractible $\left.{ }^{4}\right)$, then the cross-section theorem is valid, i.e., if $g: A \rightarrow X$ is a map from a closed subset $A$ into $X$ such that $p g=1$, then there exists an extension $\bar{g}: B \rightarrow X$ of $g$ such that $p \bar{g}=1$.

Proof. Using Proposition 5, we have a family of "coordinate neighborhoods" $\left\{U_{\alpha}\right\}$ covering $B$, a space $F$ and maps $\psi_{\alpha}: p^{-1}(U) \leftrightarrows U_{\alpha} \times F: \phi_{\alpha}$ such that $\psi_{\alpha} \phi_{\alpha} \sim 1$ and $\phi_{\alpha} \psi_{\alpha} \sim 1$ preserving projections. Since the fibers are contractible, we may assume that $F$ is a single point. Let $\left\{W_{j}\right\}$ denote a countable open cover of $X$ such that for each $j, \bar{W}_{j} \subseteq U_{\alpha}$ for some $\alpha$. Suppose that $g: A \rightarrow X$ is a given "partial" cross section where $A$ is closed in $B$. We proceed to extend the cross section to $A \cup \bar{W}_{1}=A_{1}$. This, of course, will suffice to prove the result. Fix an $\alpha$ such that $\bar{W}_{1} \subseteq U_{\alpha}$. For $b \in \bar{W}_{1}$, set $h(b)=\phi_{\alpha}(b, F)$. Then, $h: \bar{W}_{1} \rightarrow p^{-1}(U)$ is a cross section over $\bar{W}_{1}$. Let $C_{1}=A \cap \bar{W}_{1}$. We show next that $h\left|C_{1} \sim g\right| C_{1}$ in the class of cross sections. Let $\Gamma: p^{-1}\left(U_{\alpha}\right) \times I \rightarrow p^{-1}\left(U_{\alpha}\right)$ denote a homotopy such that $\Gamma_{0}=1, \Gamma_{1}=\phi_{\alpha} \psi_{\alpha}$, and $p \Gamma(x, t)=p(x)$, for $x \in p^{-1}\left(U_{\alpha}\right)$ and $t \in I$. Set

$$
\gamma(b, t)=\Gamma(g(b), t) ; \quad b \in C_{1}, t \in I .
$$

Then, for each $t, \gamma_{t}$ is a cross section on $C_{1}$. Furthermore, $\gamma_{0}=g \mid C_{1}$. Also, $\gamma(b, 1)=\Gamma(g(b), 1)=\phi_{\alpha} \psi_{\alpha}(g(b))=\phi_{\alpha}(b, F)=h(b)$. Therefore, $\gamma_{1}=h \mid C_{1}$. We now apply the homotopy extension theorem as follows. Using $h, \gamma_{1}$ : $C_{1} \rightarrow p^{-1}(U \alpha)$ has an extension to $\bar{W}_{1}$. Therefore,

$$
\gamma:\left(C_{1} \times I\right) \cup\left(\bar{W}_{1} \times\{1\}\right) \rightarrow p^{-1}\left(U_{\alpha}\right)
$$

is a partial homotopy and hence we have a homotopy extension

$$
\gamma^{*}: \bar{W}_{1} \times I \rightarrow p^{-1}\left(U_{\alpha}\right)
$$

of $\gamma$. Set $\gamma_{0}^{*}=g^{*}: \bar{W}_{1} \rightarrow p^{-1}\left(U_{\alpha}\right) . g^{*}=g$ on $C_{1}$ but $g^{*}$ is not yet a cross section. We do have, however, $p g^{*} \sim 1$ with a homotopy $H: \bar{W}_{1} \times I \rightarrow U_{\alpha}$ such that $H_{0}=p g^{*}, H_{1}=1$ and $H(b, t)=b$ for $b \in C_{1}$. Interpret $H$ as a map $H^{*}: \bar{W}_{1} \rightarrow U_{\alpha}^{I}$ and let $\lambda$ denote a regular lifting function for $(X, B, p)$. We "pull" $g^{*}$ over to a cross section as follows. Set

$$
\bar{g}(b)=\lambda\left[g^{*}(b), H^{*}(b)\right](1) .
$$

(4) The fibers, therefore, may not be solid. 
Then, $\bar{g}$ is a cross section over $\bar{W}_{1}$ which agrees with $g$ on $C_{1}$. Setting $\bar{g}(b)$ $=g(b)$ on $A$, we see that $\bar{g}: A_{1} \rightarrow X$ is the required extension of $g$ to $A_{1}$.

Remark. Proposition 9 is valid under the following hypotheses: $(X, B, p)$ a regular fiber space with $X$ an $\operatorname{ANR}(Q)$, where $Q$ contains the closed subsets of $B$ (see Hanner [8]), $B$ 0-connected, wlc, Lindelof and normal, and the fibers contractible.

We next prove the following lemma:

Lemma. Let $(X, B, p)$ denote a fiber space where $X$ is a separable metric $A N R$. If $B$ is 0-connected (equivalent to p being "onto"), wlc and paracompact, then $B$ as well as the fibers are separable metric $A N R$ 's.

Proof. We show first that $B$ is a separable metric ANR. Let $U$ denote an open set containing $b$ which is contractible in $X$ to $b$. Then, $\left(p^{-1}(U), U, p\right)$ is fiber-homotopy equivalent to $U \times F$ and hence $p$ admits a local cross section over $U$. Therefore, $U$ may be imbedded in $p^{-1}(U)$ where it is a retract of $p^{-1}(U)$. Since $p^{-1}(U)$ is an ANR, $U$ is an ANR (separable metric). Since $B$ is locally metric and paracompact, it is metric [5]. Furthermore, since $B$ is the continuous image of a separable metric space, it is separable. Finally, $B$ is a local ANR and hence an ANR (separable metric) [9]. Next, we show that any fiber $F=p^{-1}\left(b_{0}\right)$ is a neighborhood retract in $X$. Since $B$ is an ANR (separable metric) there is a neighborhood $G$ of $b_{0}$ which is contractible to $b$ in $B$ relative to $b_{0}$, i.e., there is a map $H: G \rightarrow B^{I}$ such that $H(b)(0)=b, H(b)(1)=b_{0}$ and $H\left(b_{0}\right)$ is the constant path at $b_{0}$. For $x \in p^{-1}(G)$, set

$$
r(x)=\lambda[x, H(p(x))](1),
$$

where $\lambda$ is a regular lifting function ( $B$ is metric). $r(x)$ is clearly a retraction of $p^{-1}(F)$ into $F$. Hence $F$ is an ANR.

REMARk. This lemma is false without the wlc condition on $B$. Furthermore, the corresponding theorem for fiber bundles is trivial (with no hypotheses on $B$ ). This lemma raises the following question which is easily answered for fiber bundles in the affirmative.

Question. If $(X, B, p)$ is a fiber space in which $X$ is separable metric and $B$ as well as the fibers are separable metric ANR's, is $X$ an ANR?

The final proposition in this section is an analogue of Theorem 1.2 of E. H. Spanier and J. H. C. Whitehead [6] and the proof is essentially that used by Serre in [3] and does not require proving that the fibers are $H$-spaces or any cross section theorem, nor does it require local compactness. We merely need the fact that a separable metric ANR, all of whose homotopy groups vanish, is contractible.

Proposition 10. Let $(X, B, p)$ denote a fiber space in which $X$ is a finite dimensional, separable metric $A R$. Suppose $B$ is 0-connected, paracompact, and wlc and that the fibers are 0-connected. Then, $(X, B, p)$ is fiber-homotopy equivalent to a product $B \times F$, where $F$ is any fiber. 
Proof. By the previous Lemma B and any fiber $F$ are separable metric ANR. Since $p$ admits of local cross sections ( $B$ is wlc) $B$ is finite dimensional. Of course $F$ is, clearly. Therefore, using the result in Serre [3], since $\pi_{1}(B)=0$ and $X$ is homologically trivial, we have that $B$ (and $F$ ) is homologically trivial. Since $B$ is simply connected and acyclic, $B$ is contractible. Hence by Proposition $4,(X, B, p)$ is fiber-homotopy equivalent to $B \times F$.

Remark. Proposition 10 is false without the wlc condition on $B$ (see $\$ 4$ ).

4. An example. Let $B$ denote the well-known space in the plane constructed as follows. Let $A_{1}=\{(x, y) \mid x=0,-2 \leqq y \leqq 1\}, A_{2}=\{(x, y) \mid 0 \leqq x \leqq 1$, $y=-2\}, A_{3}=\{(x, y) \mid x=1,-2 \leqq y \leqq 0\}, A_{4}=\{(x, y) \mid 0<x \leqq 1$, $y=\sin (2 \pi / x)\}$. Then, $B=\bigcup A_{i}$. Let $X$ denote the half-open interval $[0,4)$ and let $f: X \rightarrow B$ denote the "natural" biunique map taking $[0,1]$ onto $A_{1}$, $[1,2]$ onto $A_{2},[2,3]$ onto $A_{3}$ and $[3,4)$ onto $A_{4}$. Let $b_{0}=(0,1)$ and $\widetilde{B}$ the space of paths in $B$ emanating from $b_{0}$. Then $(\widetilde{B}, B, p)$ is a regular fiber space, where $p$ maps a path into its right hand end point, and the fiber over $b_{0}$ is the loop space $\Lambda$ of $B$ based at $b_{0}$. Furthermore, let $\tilde{X}$ denote the space of paths based in $X$ at 0 . Then, $f: X \rightarrow B$ induces a map $\tilde{f}: \tilde{X} \rightarrow \tilde{B}$. Let $g=f^{-1}: B \rightarrow X$, which is a function but not a map. It is a simple matter to show that $g$ induces a map $\tilde{g}: \widetilde{B} \rightarrow \tilde{X}$ which is the inverse of $\tilde{f}$. Hence, $\widetilde{B}$ and $\Lambda$ are AR's, whereas $B$ is not. This serves as a counter-example for the following results in $\$ 3$.

(a) Propositions 4 and 5. The validity of these propositions implies the existence of local cross sections which would imply that $B$ is a local ANR and hence an ANR.

(b) Proposition 9. The validity of this proposition would imply that $B$ is an ANR.

(c) The lemma preceding Proposition 10.

In fact, in the above $(X, B, f)$ is itself a fibering in the sense of Hurewicz. This shows that the theorem of Whitehead-Spanier (Proposition 10) is false for fiber spaces without the wlc assumption on $B$.

5. A definition of fiber space, invariant under fiber-homotopy equivalence. The definition of equivalent fiber bundles (=locally trivial) usually employs a fiber-preserving homeomorphism. It is a trivial result that if $(X, B, p),(Y, B, q)$ are triples and if $f: X \rightarrow Y$ is a fiber-preserving homeomorphism, then if $(X, B, p)$ is a fiber bundle so is $(Y, B, q)$. The corresponding theorem for fiber spaces is false if one uses fiber-homotopy equivalence and the usual definitions of fiber spaces (with the exception, of course, of that employed by A. Grothendieck). In this section, we explore a definition of fiber space which is very similar to the definition of fiber bundle and which further

(1) is invariant under fiber-homotopy equivalence,

(2) yields a covering homotopy theorem which is sufficient to imply the exactness of the homotopy sequence and also the Leray-Serre theorem for the associated spectral sequence,

(3) yields a cross-section theorem when the fibers are contractible.

The definition, which is quite natural, is as follows. 
Definition. Let $p: X \rightarrow B$ denote a map and $F$ a space such that there exists an open cover $\left\{U_{\alpha}\right\}$ of $B$ with $p^{-1}\left(U_{\alpha}\right)$ fiber-homotopy equivalent to $U_{\alpha} \times F$. Then $(X, B, p)$ is called a fiber space.

In short, a fiber space is a local product space-up to fiber-homotopy equivalence. It is immediate that this definition is invariant under fiberhomotopy equivalence and that all fiber bundles are fiber spaces in this sense. Furthermore, if $(X, B, p)$ is a fiber space in the sense of Hurewicz ( $\$ 2)$, where $B$ is 0 -connected and wlc, then $(X, B, p)$ is a fiber space. (It is understood that we use the term fiber space in the above sense in the rest of the paper.) Also, it is immediate from the definition of fiber space above that all fibers $p^{-1}(b), b \in B$ are of the same homotopy type. We proceed now to prove a covering homotopy theorem for fiber spaces and begin with some definitions. This theorem is complicated by the fact that one may not even be able to lift arcs in the usual manner. For example, let $X$ be the set of points in the plane $A \cup B$ where $A=\{(1 / 2, y) \mid-1 \leqq y \leqq 1\}$ and $B=\{(x, 0) \mid 0 \leqq x \leqq 1\}$. Then $(X, B, p)$ is a fiber space, where $p$ is the natural projection. However, one cannot lift arcs in $B$ which begin at the "singular point" $(1 / 2,0)$, with preassigned initial point.

Definition. Let $H: Y \times I \rightarrow B, G: Y \times I \rightarrow B$ denote given homotopies. Then $H$ and $G$ are said to be strongly homotopic if there exists a map $\Gamma$ : $Y \times I \times I \rightarrow B$ such that

(1) $\Gamma(y, t, 0)=H(y, t), \Gamma(y, t, 1)=G(y, t) ; y \in Y, 0 \leqq t \leqq 1$,

(2) $\Gamma(y, 0, s)=H(y, 0), \Gamma(y, 1, s)=H(y, 1) ; y \in Y, 0 \leqq s \leqq 1$,

(3) if for fixed $y \in Y, H(y, t)$ is stationary with $t$, then $\Gamma(y, t, s)$ is stationary with $t$ and $s$.

Generalized Covering homotopy theorem $(g C H T)$. Let $(X, B, p) d e-$ note a triple, $p: X \rightarrow B$, and $Y$ a topological space. Then, the gCHT is said to hold for $(X, B, p)$ wrt $Y$ if whenever $H: Y \times I \rightarrow B$ and $g: Y \rightarrow X$ are given maps such that $p g=H_{0}$, then there exists a homotopy $G: Y \times I \rightarrow X$ such that $G_{0}=g$ and $H$ is strongly homotopic to $p G$.

Theorem 1. Let $(X, B, p)$ denote a fiber space. Then, the gCHT holds for $(X, B, p)$ wrt compact Hausdorff spaces.

Proof. The proof is a modification of that in Steenrod [7] for bundles. Let $\left\{U_{\alpha}\right\}$ denote an open cover of $B$ such that $\left(p^{-1}\left(U_{\alpha}\right), U_{\alpha}, p\right)$ is fiberhomotopy equivalent to $U_{\alpha} \times F$, and let $\psi_{\alpha}: p^{-1}\left(U_{\alpha}\right) \rightleftarrows U_{\alpha} \times F: \phi_{\alpha}$, denote a fiber-homotopy equivalence. Now, suppose $Y$ is a compact Hausdorff space, $H: Y \times I \rightarrow B$ is a given homotopy and $g: Y \rightarrow X$ is a map covering the initial stage of $H$, i.e., $p g=H_{0}$. Then, $H^{-1}\left(U_{\alpha}\right)$ is an open cover of $Y \times I$ and, consequently, we have a finite refinement of the form $\left\{V_{j} \times I_{k}\right\}$ where $V_{j}, j$ $=1, \cdots, m$, are open sets in $Y$ and $I_{k}, k=1, \cdots, n$, are intervals, open in $I$, such that $I_{k} \cap I_{k^{\prime}} \neq \phi$ if and only if $k-k^{\prime}= \pm 1$. For each $k, 1 \leqq k \leqq n-1$, fix a point $t_{k} \in I_{k} \cap I_{k+1}$, and let $t_{0}=0$ and $t_{n}=1$. Then, $0=t_{0}<t_{1}<\cdots<t_{n}=1$. Now, for each $y \in Y$ select a triple of open sets $W, W^{\prime}, W^{\prime \prime}$, such that 


$$
y \in W \subseteq \bar{W} \subseteq W^{\prime} \subseteq \bar{W}^{\prime} \subseteq W^{\prime \prime} \subseteq \bar{W}^{\prime \prime} \subseteq V_{j} \text { for some } j .
$$

A finite number of these triples, $W_{i}, W_{i}^{\prime}, W_{i}^{\prime \prime}, 1 \leqq i \leqq s$, can be chosen so that $U W_{i}=Y$. We proceed to "cover" $H: Y \times\left[t_{0}, t_{1}\right] \rightarrow B$. Let $u_{i}: Y \rightarrow\left[t_{0}, t_{1}\right]$ denote Urysohn maps such that

$$
u_{i}\left(\bar{W}_{i}\right)=t_{1}, \quad u_{i}\left(Y-W_{i}^{\prime}\right)=t_{0} .
$$

Furthermore, define maps $\tau_{i}: Y \rightarrow[0,1]$ as follows $\tau_{0}=0$ and for $i \geqq 1$ set

$$
\tau_{i}(y)=\max u_{1}(y), \cdots, u_{i}(y) .
$$

Then, $0=\tau_{0}(y) \leqq \tau_{1}(y) \leqq \tau_{2}(y) \leqq \cdots \leqq \tau_{s}(y)=t_{1}$, for $y \in Y$. We now proceed to define a map $G^{1}: Y \times\left[t_{0}, t_{1}\right] \rightarrow X$ which "almost" covers $H: Y \times\left[t_{0}, t_{1}\right] \rightarrow B$. As a matter of convenience, we will work with the interval $\left[t_{0}, 2 t_{1}-t_{0}\right]$ and "shrink it" later. We decompose $Y \times\left[t_{0}, 2 t_{1}-t_{0}\right]$ into closed sets as follows: For, $1 \leqq i \leqq s$, and $\lambda=\left(t_{1}-t_{0}\right) / s$

$$
\begin{aligned}
& Z_{i}=\left\{(y, t) \mid y \in Y, \tau_{i-1}(y)+(i-1) \lambda \leqq t \leqq \tau_{i-1}(y)+i \lambda\right\}, \\
& \left.Y_{i}=\{y, t) \mid y \in \bar{W}_{i}^{\prime}, \tau_{i-1}(y)+i \lambda \leqq t \leqq \tau_{i}(y)+i \lambda\right\} .
\end{aligned}
$$

Then, if we set $K_{i}=Z_{i} \cup Y_{i}$, it is clear that

$$
K_{1} \subseteq K_{2} \subseteq \cdots \subseteq K_{s}=Y \times\left[t_{0}, 2 t_{1}-t_{0}\right]
$$

We define $G_{*}^{1}: K_{1} \rightarrow X$ as follows. Consider $(y, t) \in Y_{1}$, whence $y \in \bar{W}_{1}^{\prime}$ and $\tau_{0}(y)+\lambda \leqq t \leqq \tau_{1}(y)+\lambda$. Set

$$
G_{*}^{1}(y, t)=\phi_{\alpha}\left[H(y, t-\lambda), \psi_{\alpha}(g(y))_{2}\right], \quad(y, t) \in Y_{1}
$$

where $H\left(\bar{W}_{1}^{\prime} \times\left[t_{0}, t_{1}\right]\right) \subseteq U_{\alpha}$ and $\psi_{\alpha}(g(y))_{2} \in F$ is the second coordinate of $\psi_{\alpha}(g(y)) . G_{*}^{1}$ is extended to $Z_{1}$ as follows, $\phi_{\alpha} \psi_{\alpha} \sim 1$ preserving projections. Let $\Gamma: p^{-1}\left(U_{\alpha}\right) \times I \rightarrow p^{-1}\left(U_{\alpha}\right)$ denote a projection-preserving homotopy such that $\Gamma_{0}=1$, and $\Gamma_{1}=\phi_{\alpha} \psi_{\alpha}$. Also, let $u_{1}^{\prime}$ denote a Urysohn map, $u_{1}^{\prime}: Y \rightarrow[0,1]$ such that

$$
u_{1}^{\prime}\left(\bar{W}_{1}^{\prime}\right)=1 \text { and } u_{1}^{\prime}\left(Y-W_{1}^{\prime \prime}\right)=0 .
$$

Set

$$
G_{*}^{1}(y, t)=\Gamma\left[g(y), u_{1}^{\prime}(y) \frac{t}{\lambda}\right], \quad(y, t) \in Z_{1} .
$$

Now, take $(y, t) \in Y_{1} \cap Z_{1}$. Then, $y \in \bar{W}_{1}^{\prime}$ and $t=\lambda$, whence using either (1) or (2), we obtain

$$
G_{*}^{1}(y, t)=\phi_{\alpha} \psi_{\alpha}(g(y)), \quad(y, t) \in Y_{1} \cap Z_{1} .
$$

Therefore, $G_{*}^{1}$ is defined and continuous on $K_{1}$. We note the following properties of $G_{*}^{1}$ : 
(i) $p G_{*}^{1}(y, t)=p g(y)=H(y, 0),(y, t) \in Z_{1}$,

(ii) $p G_{*}^{\mathbf{1}}(y, t)=H(y, t-\lambda),(y, t) \in Y_{1}$.

Now, let

$$
g_{1}(y)=G_{*}^{1}\left(y, \tau_{1}(y)+\lambda\right), \quad y \in Y .
$$

We now define $G_{*}^{2}: K_{2} \rightarrow X$. Take $\left(y_{0}, t\right) \in Y_{2}$ and set

$$
G_{*}^{2}(y, t)=\phi_{\beta}\left[H(y, t-2 \lambda), \psi_{\beta}\left(g_{1}(y)\right)_{2}\right], \quad(y, t) \in Y_{2}
$$

where $H\left(\bar{W}_{2}^{\prime} \times\left[t_{0}, t_{1}\right]\right) \subseteq U_{\beta}$ for some index $\beta$. Now, let $\Gamma^{\prime}: p^{-1}\left(U_{\beta}\right)$ $\times I \rightarrow p^{-1}\left(U_{\beta}\right)$ denote a projection-preserving homotopy such that $\Gamma_{0}^{\prime}=1$ and $\Gamma_{1}^{\prime}=\phi_{\beta} \psi_{\beta}$. Also, let $u_{2}^{\prime}$ denote a Urysohn map $u_{2}^{\prime}: Y \rightarrow[0,1]$ such that

$$
u_{2}^{\prime}\left(\bar{W}_{2}^{\prime}\right)=1 \text { and } u_{2}^{\prime}\left(Y-W_{2}^{\prime \prime}\right)=0 .
$$

Set

$$
G_{*}^{2}(y, t)=\Gamma^{\prime}\left[g_{1}(y), u_{2}^{\prime}(y) \frac{\left(t-\tau_{1}(y)-\lambda\right)}{\lambda}\right], \quad(y, t) \in Z_{2} .
$$

We check first, the consistency of $G_{*}^{2}$ on $Z_{2} \cap Y_{2}$. Take $(y, t) \in Z_{2} \cap Y_{2}$. Then $y \in \bar{W}_{2}^{\prime}$ and $t=\tau_{1}(y)+2 \lambda$. Then, using (3)

$$
G_{*}^{2}(y, t)=\phi_{\beta}\left[H\left(y, \tau_{1}(y)\right), \psi_{\beta}\left(g_{1}(y)\right)_{2}\right]=\phi_{\beta} \psi_{\beta}\left(g_{1}(y)\right) .
$$

Furthermore, using (4)

$$
G_{*}^{2}(y, t)=\Gamma^{\prime}\left[g_{1}(y), 1\right]=\phi_{\beta} \psi_{\beta} g_{1}(y) .
$$

Therefore, $G_{*}^{2}(y, t): K_{2} \rightarrow X$ is a well-defined map. We note the following properties of $G_{*}^{2}$ :

(i) $p G_{*}^{2}(y, t)=p g_{1}(y)=H\left(y, \tau_{1}(y)\right),(y, t) \in Z_{2}$,

(ii) $p G_{*}^{2}(y, t)=H(y, t-2 \lambda),(y, t) \in Y_{2}$,

(iii) $G_{*}^{2}(y, t)=G_{*}^{1}(y, t)$ for $(y, t) \in K_{1} \cap K_{2}$.

We verify (iii) as follows. It suffices to consider $(y, t) \in K_{1} \cap Z_{2}$. In such a case it is easy to see that $t=\tau_{1}(y)+\lambda$ and hence

$$
G_{*}^{1}(y, t)=G_{*}^{1}\left(y, \tau_{1}(y)+\lambda\right)=g_{1}(y)=\Gamma^{\prime}\left[g_{1}(y), 0\right]=G_{*}^{2}(y, t) .
$$

Hence, $G_{*}^{1}=G_{*}^{2}$ on $K_{1} \cap K_{2}$.

Continuing in the obvious manner we obtain maps $G_{*}^{t}: K_{i} \rightarrow X, 1 \leqq i \leqq s$ with the following properties:

(i) $p G_{*}^{i}(y, t)=H\left(y, \tau_{i-1}(y)\right),(y, t) \in Z_{i}$,

(ii) $p G_{*}^{i}(y, t)=H(y, t-i \lambda),(y, t) \in Y_{i}$,

(iii) $G_{*}^{*}(y, t)=G_{*}^{*}-1(y, t),(y, t) \in K_{i} \cap K_{i-1}$.

Thus, we may define a homotopy $G_{*}: Y \times\left[t_{0}, 2 t_{1}-t_{0}\right] \rightarrow X$ by setting

$$
G_{*}(y, t)=G_{*}^{i}(y, t)
$$


where $(y, t) \in K_{i}$. Define $G^{1}: Y \times\left[t_{0}, t_{1}\right] \rightarrow X$ by

$$
G^{1}(y, t)=G_{*}\left(y, 2 t-t_{0}\right) .
$$

Now it is clear that $G^{1}\left(y, t_{0}\right)=g(y)$, and we show next that $p G^{1}$ is strongly homotopic to $H: Y \times\left[t_{0}, t_{1}\right] \rightarrow B$.

We define a homotopy $\Phi: Y \times\left[t_{0}, t_{1}\right] \times I \rightarrow B$ as follows. Take $(y, t) \in Y$ $\times\left[t_{0}, t_{1}\right]$. Set

(a) $\Phi(y, t, s)=H\left[y, t(1-s)+\tau_{i-1}(y) s\right]$ if

$$
\frac{\tau_{i-1}(y)+(i-1) \lambda+t_{0}}{2} \leqq t \leqq \frac{\tau_{i-1}(y)+i \lambda+t_{0}}{2},
$$

(b) $\Phi(y, t, s)=H\left[y, t(1-s)+s\left(2 t-i \lambda-t_{0}\right)\right]$ if

$$
\frac{\tau_{i-1}(y)+i \lambda+t_{0}}{2} \leqq t \leqq \frac{\tau_{i}(y)+i \lambda+t_{0}}{2} .
$$

We note that $\Phi$ is a well-defined map and list its properties which are easily verified

(1) $\Phi(y, t, 0)=H(y, t), \Phi(y, t, 1)=p G^{1}(y, t)$,

(2) $\Phi\left(y, t_{0}, s\right)=H\left(y, t_{0}\right), \Phi\left(y, t_{1}, s\right)=H(y, t)$,

(3) If $H(y, t)$ is stationary with $t$, for fixed $y$, then $\Phi(y, t, s)$ is stationary with $t$ and $s$.

Thus, we see that $p G^{1}: Y \times\left[t_{0}, t_{1}\right] \rightarrow B$ is strongly homotopic to $H$. Now, working successively with the intervals $\left[t_{1}, t_{2}\right],\left[t_{2}, t_{3}\right], \cdots$ we obtain a homotopy $G: Y \times[0,1] \rightarrow B$ such that $p G$ is strongly homotopic to $H$ and our proof is complete.

We mention the following extension of the above theorem. The proof involves fitting homotopies together in an uninteresting fashion and is omitted.

Theorem. Let $(X, B, p)$ denote a fiber space. Then, the gCHT holds for $(X, B, p)$ wrt $C_{o}$-spaces [7].

REMARk. One should be able to adopt the techniques used by Hurewicz [1] to prove a universal gCHT if $B$ is paracompact. A possible approach would be to ask whether given a fiber space $(X, B, p)$, does there exist a fiber space $(Y, B, q)$ in the sense of Hurewicz ( $\$ 2)$ which is fiber-homotopy equivalent to $(X, B, p)$. An affirmative answer to this question would prove the above universal gCHT.

We next prove the following cross-section theorem.

Theorem. Let $(X, B, p)$ be a fiber space with $X$ an $A N R(Q)$, where $Q$ contains the closed subsets of $B$. Suppose further that the fiber $F$ is contractible and $B$ is compact Hausdorff. Then, if $f: A \rightarrow X$ is a given cross section, $A$ closed in $B$, there exists a cross section $g: B \rightarrow X$ such that $g \mid A \sim f$ preserving projections.

Proof. Let $\left\{U_{\alpha}\right\}$ denote an open cover of $B$ such that $p^{-1}\left(U_{\alpha}\right)$ is fiber- 
homotopy equivalent to $U_{\alpha} \times F$ and let $\psi_{\alpha}$ : $p^{-1}\left(U_{\alpha}\right) \rightleftarrows U_{\alpha} \times F$ : $\phi_{\alpha}$ denote a fiber-homotopy equivalence. Furthermore, let $V_{j}, j=1, \cdots, n$ denote a finite open cover of $B$ such that $\bar{V}_{j} \subseteq U_{\alpha}$ for some $\alpha$. Suppose that $f: A \rightarrow X$ is a given cross section, where $A$ is closed in $B$. We obtain a cross section $g_{1}: A \cup \bar{V}_{1} \rightarrow X$ such that $g_{1} \mid A \sim f$ preserving projections. Since we are assuming $F$ contractible, we may assume $F$ is a single point. Fix an index $\alpha$ such that $\bar{V}_{1} \subseteq U_{\alpha}$. A cross section over $U_{\alpha}$ is obtained by setting $g_{1}(b)$ $=\phi_{\alpha}(b, F)$. Let $C=\bar{V}_{1} \cap A$. Define $G: C \times I \rightarrow X$ by

$$
G(b, t)=\Gamma(f(b), t)
$$

where $\Gamma$ is a projection-preserving homotopy: $p^{-1}\left(U_{\alpha}\right) \times I \rightarrow p^{-1}\left(U_{\alpha}\right)$ such that $\Gamma_{0}=1$ and $\Gamma_{1}=\phi_{\alpha} \psi_{\alpha}$. Then $G_{0}=f \mid C$ and $G_{1}=\phi_{\alpha} \psi_{\alpha} f \mid C$. But, for $b \in C$

$$
\phi_{\alpha} \psi_{\alpha} f(b)=\phi_{\alpha}(b, F)=g_{1}(b) .
$$

Therefore, $G_{1}=g_{1} \mid C$. We now apply the homotopy extension theorem and extend $G$ to $\bar{V}_{1} \times I$ with $G_{1}=g_{1}$. Let $H=p G$. Extend $H$ to $A \cup \bar{V}_{1} \times I$ by setting $H(b, t)=b$ for $b \in A$. Now, we apply the gCHT using $H: A \cup \bar{V}_{1} \times I \rightarrow B$ and initial map $g^{*}$ which is $f$ on $A$ and $G_{0}$ on $\bar{V}_{1}$. Therefore, there exists a homotopy $G^{*}: A \cup \bar{V}_{1} \times I \rightarrow X$ such that $p G^{*}$ is strongly homotopic to $H$ and $G_{0}^{*}=g^{*}$. Let $g=G_{1}^{*}$. Then, $g$ is a cross section over $A \cup \bar{V}_{1}$ and $g \mid A \sim f$ where the connecting projection-preserving homotopy is just $G^{*} \mid A \times I$. We then continue this procedure adding one $\bar{V}_{j}$ at a time to the range the cross section and since the $V_{j}$ are finite in number our result follows.

Theorem. The above cross-section theorem holds for base spaces which are $C_{\sigma}$ and $A$ is compact.

Proof. By assumption, we have a countable sequence of open sets $W_{i}$ such that $\bar{W}_{i}$ are compact, $\bar{W}_{i} \subseteq W_{i+1}$ and $\cup W_{i}=B$. We may assume without loss that $A \subseteq W_{1}$. Now, let $f: A \rightarrow X$ denote the given cross section and let $u_{k}$, $k=0,1,2, \cdots$ denote Urysohn maps $B \rightarrow[0,1]$ such that

$$
u_{k}\left(\bar{W}_{k-1}\right)=0, \quad u_{k}\left(Y-W_{k}\right)=1
$$

where we set $W_{0}=A$ for notational convenience.

Let $g_{1}$ denote a cross section over $\bar{W}_{1}$ such that $g_{1} \mid A \sim f$, preserving projections, and in general, let $g_{k}$ denote a cross section over $\bar{W}_{k}$ such that $g_{k} \mid \bar{W}_{k-1}$ $\sim g_{k-1}$, preserving projections. Furthermore, let $\Gamma^{k}: \bar{W}_{k} \times I \rightarrow X$ denote a projection-preserving homotopy such that $\Gamma_{0}^{k}=g_{k}$ and $\Gamma_{1}^{k}=g_{k+1} \mid \bar{W}_{k}$. For $b \in \bar{W}_{k}-W_{k-1}, k \geqq 1$, set

$$
g(b)=\Gamma^{k}\left(b, u_{k}(b)\right) .
$$

For $b \in W_{0}$, set $g(b)=g_{1}(b) . g$ is clearly a cross section and $g \mid A \sim f$, preserving projections, which completes the proof.

REMARK. The reader will note that we have required that the base space be locally compact in the above theorem. This restriction is not made in the 
classical cross section theorem for bundles [7] nor in Proposition 9 of $\$ 3$.

Added in proof. With regard to properties (1), (2), and (3) mentioned in the first paragraph of this section, it should be remarked that the definition of fiber space employed here also satisfies

(4) if $(X, B, p)$ is a fiber space and $f: A \rightarrow B$ is a map, then $\left(f^{-1}(X), q, A\right)$ is also a fiber space, where $f^{-1}(X)=\{(a, x) \in A \times X \mid f(a)=p(x)\}$ and $q(a, x)$ $=a$.

This property (4) is not enjoyed by the quasi-fibrations of Dold and Thom [10].

\section{BIBLIOGRAPHY}

1. W. Hurewicz, On the concept of fiber space, Proc. Nat. Acad. Sci. U.S.A. vol. 41 (1955) pp. $956-961$.

2. R. Thom, Espaces fibres et carres de Steenrod, Ann. Sci. École Norm. Sup. (1952) pp. 109-182. 505.

3. J. P. Serre, Homologie singuliere des espaces fibres, Ann. of Math. vol. 54 (1951) pp. 425-

4. G. S. Young, On the factors and fiberings on manifolds, Proc. Amer. Math. Soc. vol. 1 (1950) pp. 215-223. no. 91 .

5. Y. M. Smirnov, On metrization of topolcgical spaces, Amer. Math Soc. Translations,

6. J. H. C. Whitehead and E. H. Spanier, On fiber spaces in which the fiber is contractible, Comment. Math. Helv. vol. 29 (1955) pp. 1-7.

7. N. Steenrod, The topology of fiber bundles, Princeton University Press.

8. O. Hanner, Retraction and extension of mappings of metric and non-metric spaces. Ark. Mat. vol. 2 (1952) pp. 315-360.

9. - Some theorems on ANR's, Ark. Mat. vol. 1 (1951) pp. 389-408.

10. A. Dold and R. Thom, Quasifaserugen und unendliche symmetrische Producte, Ann. of Math. vol. 67 (1958) pp. 239-281.

UNIVERSITY OF WISCONSIN, MAdison, Wis. 